\title{
Xoob: observaciones sobre la producción de silbatos en Uayma, Yucatán
}

\section{Xoob: Observations on the Production of Whistles in Uayma, Yucatan}

\author{
Pedro Rogelio Xuluc Balam \\ Instituto Nacional de Antropología e Historia, Centro Yucatán, México
}

\begin{abstract}
Resumen: Este trabajo busca una comprensión a la labor de manufactura de silbatos cerámicos en la comunidad de Uayma, Yucatán. Como parte de los objetivos se documentaron las características tangibles e intangibles que involucran el proceso de creación de seis aerófonos seleccionados de una muestra mayor de doce artefactos sonoros. La investigación enmarca los vínculos de la cultura material, el conocimiento y la tecnología empleada en la fabricación de aerófonos, así como la cosmovisión de los productores. Se presentan los resultados de la morfología, acústica y funcionalidad de estos artefactos a través de un estudio de imagenología de rayos $\mathrm{X}$ aplicada a la muestra para poder entender las variantes existentes en las formas interna y externa que regulan las condiciones de ejecución, así como la acústica y funcionalidad del instrumento. El presente estudio se suma, como una opción interpretativa, a las múltiples propuestas derivadas de la temática de producción de artefactos sonoros en cerámica para el área maya.
\end{abstract}

Palabras clave: silbatos; aerófonos; Uayma; producción cerámica; rayos X.

ABSTRACT: This work seeks an understanding of the elaboration of ceramic whistles in the community of Uayma, Yucatan. As part of the objectives, the tangible and intangible characteristics that involve the process of creating six selected aerophones from a larger sample of twelve sound artifacts were documented. The research frames the links of material culture, knowledge and technology used in the manufacture of aerophones, as well as the worldview of producers. The results of the morphology, acoustics and functionality of these artifacts are presented through an X-ray imaging study applied to the sample in order to understand the variants existing in the internal and external forms that regulate the conditions of execution, as well as the acoustics and functionality of the instrument. The present study 
adds, as an interpretive option, to the multiple proposals derived from the theme of production of ceramic sound artifacts for the Maya area.

KEYwORDS: whistles; aerophones; Uayma; ceramic production; X-rays.

RECEPCIÓN: 23 de octubre de 2019.

ACEPTACIÓN: 9 de diciembre de 2019.

Dor: https://doi.org/10.19130/iifl.ecm.2020.56.2.0003

\section{Introducción}

La comprensión del aspecto material es fundamental para complementar el panorama de investigación donde los fenómenos sociales del pasado fueron parte de un amplio universo de oficios, trabajos y acciones que vinculaban lo ritual con lo práctico o simplemente lo tradicional con lo innovador (Hodder, 1991). Los estudios de manufactura en arqueología han permitido observar la vida entera de una clase de artefactos, desde evidenciar la obtención de la materia prima, distinguir entre áreas de actividad y describir las razones por las cuales un grupo de alfareros explotaba varios yacimientos diferentes, además de entender cómo se deposita el material de descarte, estudiar el entorno de manufactura y los factores modificantes del mismo; estos son algunos ejemplos que ofrece el análisis de la producción en el pasado (Costin, 1991, 2001; Deal, 1983: 41; 1998: 32).

La producción de algún artefacto requiere de decisiones, ${ }^{1}$ y el conocimiento de la materia prima, herramientas, técnicas y acabados es la mejor manera de definir el proceso de manufactura exclusivo de algún artículo. La secuencia operacional y las selecciones particulares serán la convergencia hacia opciones favorables en su elaboración (Sillar, 2000). Las decisiones o elecciones son influenciadas por alteraciones de propiedades culturales que se asocian a un rasgo social, económico o ideológico (Sinopoli, 1991: 102; Sillar, 2000); estas dinámicas serán las que establezcan la actividad relacionando la productividad, eficacia, organización interna de quien produce y ejecución de las técnicas aplicadas a la materia prima en su transformación (Gosselain y Livingstone, 2005). De acuerdo con Polanyi (1968: 137-139): “[...] producción es cualquier actividad que utiliza recursos con el objetivo de crear o proporcionar bienes, y ponerlos a disposición, cuando tal actividad constituye una condición necesaria o deseable para la disponibilidad". Es decir, "[...] que la producción implica y supone necesariamente la apropiación y transformación de recursos naturales [...] por medio de diversos instrumentos de trabajo, tanto para su obtención como para su manufactura" (Jover, 1999: 54); por lo tanto un sistema de producción desencadenará huellas en el registro arqueológico.

\footnotetext{
${ }^{1}$ Según Sillar (2000: 45), serían las siguientes: 1) materia prima para elaborar artefactos, 2) herramientas usadas para la extracción de la materia prima, 3) elementos usados para la transformación de la materia prima y 4) técnicas planteadas para trabajar la materia prima con un fin particular.
} 


\section{Antecedentes de producción alfarera en el norte de Yucatán}

La alfarería se destaca por ser uno de los primeros oficios de la humanidad y se ha desarrollado a través de diversas culturas y regiones. El trabajo alfarero presenta etapas en que los artesanos optimizan las mejores decisiones para su producción, establecen tiempos en su manufactura y los vinculan en su proceso de creación; las etapas generales del proceso alfarero son la recolección de la materia prima, preparación, molido, mezclado y amasado, secado, modelado, bruñido-decorado-pintado, cocción y en casos algún tratamiento post cocción (Orton, 1997: 133; Rice, 1996: 173).

En el área maya, la arqueología ha rastreado elementos de este oficio para las diversas zonas que comprende la cultura en su pasado prehispánico, histórico y contemporáneo. Las primeras investigaciones sobre la producción alfarera en Yucatán se remontan a mediados del siglo pasado. Raymond Thompson (1958) fue uno de los investigadores que más detalle generó en sus registros sobre la alfarería peninsular (Figura 1). En otros datos publicados en la Enciclopedia Yucatanense por Hernández (1977), se realiza una reseña de la alfarería del Estado, resaltando la mención de "minas" (bancos de material) de barro en las localidades de Ticul, Maxcanú, Valladolid, Tizimín y Chikindzonot.

Según refieren Rendón (1947), Varela (1990) y Morales (2005) existen tres tipos de alfarería maya yucateca: de uso cotidiano, ceremonial y de distribución comercial:

a) La de uso cotidiano comprende aquellos recipientes que son usados para contener agua y alimentos, así como objetos útiles y prácticos en alguna unidad doméstica.

b) La ceremonial alcanza elementos como cajetes, incensarios, candeleros, juguetes, silbatos, flautas, ocarinas y tambores que se colocan en los altares de hanal pixaan ${ }^{2}$ o en actividades rituales relacionadas con las abejas (Rosales y Rubio, 2010) y la agricultura, así como para festividades del culto católico.

c) La de carácter comercial, por su parte, es la que se distribuye al interior y en estados vecinos.

Terán y Rasmussen (1981) reportaron que en los pueblos alfareros de Yucatán se modela a mano y que Ticul se dedicaba a la producción comercial. En cuanto a los instrumentos musicales, se refiere que la mayoría de ellos eran silbatos, y que se empleaban en rituales como el cha' chak, ${ }^{3}$ los días de muertos (Maxcanú, Becal

\footnotetext{
2 En Yucatán, es el nombre que se da a la conmemoración del día de muertos, en varios lugares de la península inicia el 31 de octubre con la llegada de los difuntos infantes y concluye el 8 de noviembre con la octava de difuntos. Los términos en lengua maya se han tomado de Barrera Vásquez (1980).

${ }^{3}$ El cha'a chak es una ceremonia agrícola maya de petición de lluvia que por lo general se realiza a mitad del ciclo agrícola y durante la canícula.
} 


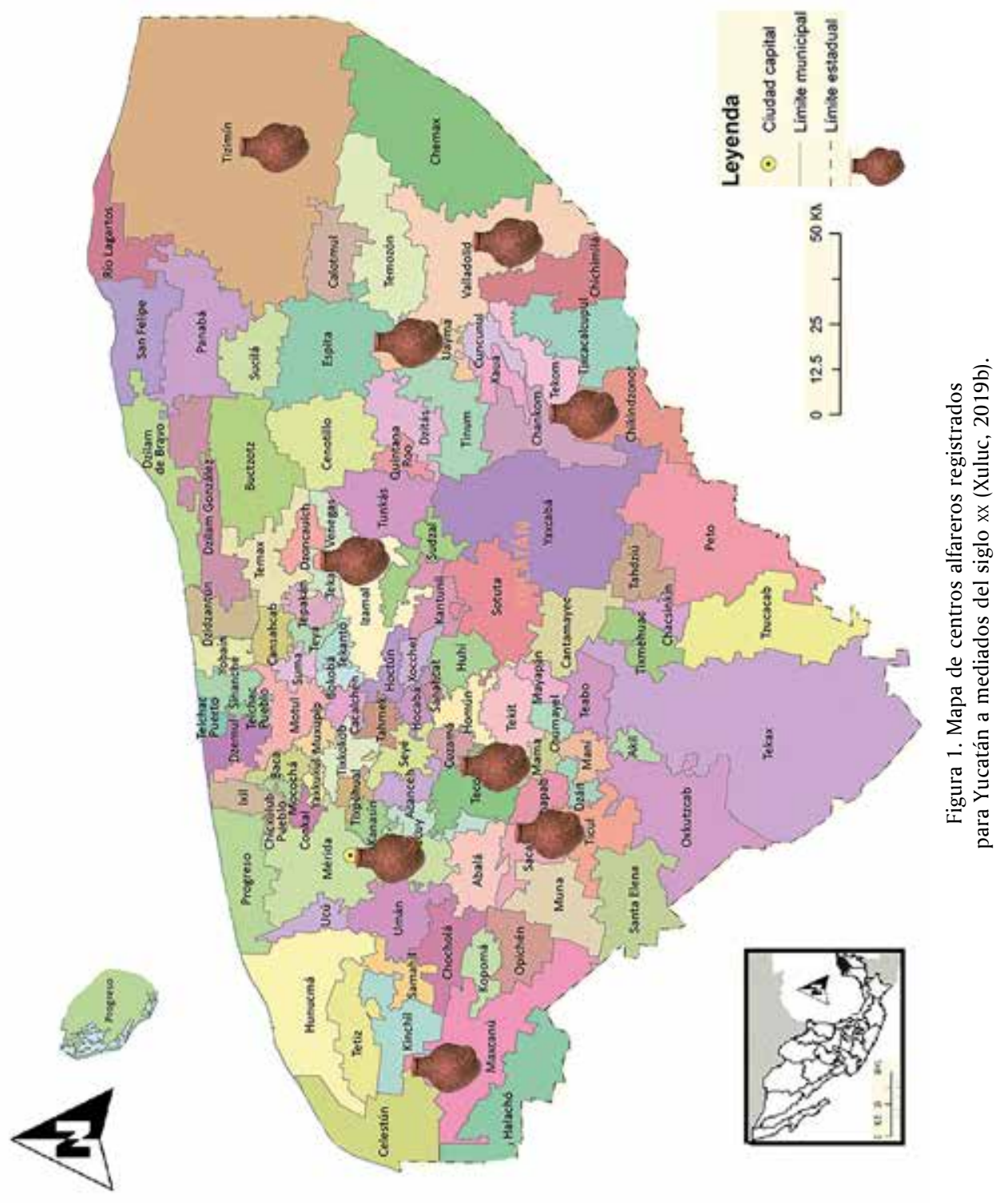


y Tepakán) (Varela, 1990) y como encargos para festividades religiosas, como las vaquerías $^{4}$ y los bailes de ramillete. ${ }^{5}$

Ya en la primera década de este siglo, Morales (2005) registró los centros alfareros contemporáneos, y observó que entre estos talleres de la península se mantienen diferencias en sus producciones y composiciones del material, técnicas y herramientas para realizar el trabajo, además de la persistencia de ciertas piezas como los cántaros ( $p^{\prime}$ úul), los apastes (k'at) y las tinajas; éstas presentan diferencias perceptibles en el color y la forma, al generar cada alfarero sus elementos cerámicos dentro de una tradición tecnológica particular de cada pueblo dedicado a la alfarería (Morales, 1992).

Los diversos elementos etnoarqueológicos y arqueológicos recuperados en el área mesoamericana delatan la importancia del estudio de las áreas de producción y los elementos relacionados con la manufactura; de ellos dependerá la formulación de hipótesis y analogías para la interpretación de la evidencia material (Andrews, 1973; Mata, 2006; Castillo et al., 2009; Zalaquett, Sierra y Jiménez, 2013).

\section{La alfarería uaymense}

La alfarería engloba un sistema amplio de relaciones tanto sociales como de técnicas y métodos basados en el entorno físico que rodea al artesano. La desarrollada en Uayma, Yucatán, fue documentada en detalle por el arqueólogo Raymond Thompson en 1958; él destaca la producción alfarera del poblado con un número determinado de familias dedicadas a la alfarería, así como de otros nueve centros vigentes en la península de Yucatán, y resaltó los conocimientos de producción y las dinámicas familiares que llevan una organización en el trabajo (Thompson, 1958).

En la actualidad el número de alfareros se ha limitado y sólo se registra el caso de la familia Espadas Dzul, que son los herederos de una tradición en la producción de artefactos cerámicos del poblado (García, 2015; Morales, 2005; Xuluc, 2019a). De acuerdo con las memorias de la familia, los conocimientos alfareros les fueron transmitidos por Juanita Dzul. ${ }^{6}$ La producción cerámica en la comunidad se remonta a seis generaciones antes de Juanita (Xuluc, 2019b) y, según afirman las fuentes orales, esta actividad relaciona el entorno con las propiedades físicas del hombre, es decir que la especialización que cada artesano va adquiriendo está vinculada a su relación con la arcilla.

\footnotetext{
${ }^{4}$ Las vaquerías son bailes tradicionales realizados durante las fiestas patronales de los pueblos mayas de la península de Yucatán.

${ }^{5}$ El ramillete es un baile llevado a cabo por los gremios durante las fiestas patronales de los pueblos mayas peninsulares.

${ }^{6}$ Ver Xuluc 2019b. En esta tesis se hace mención de las seis generaciones de maestros alfareros previas a Juanita Dzul.
} 
Con referencia a las fuentes verbales de los moradores del poblado se cuenta que los alfareros se reunían en el taller de la familia Dzul, trabajaban el barro y, más tarde, después de la cocción, mandaban a los jóvenes a vender las mercancías a rancherías, comunidades y municipios aledaños. La distribución de la venta era establecida por rutas conocidas entre los vendedores, y cada cual abarcaba cierta área llegando a caminar hasta $22 \mathrm{~km}$ diarios con la mercancía sobre los hombros y espalda.

La organización del taller ha pasado de generación en generación con un maestro alfarero al frente de los demás; en este caso, al ser el que más conocimientos heredó y aprendió, don Emilio es quien ejecuta las órdenes que conciernen a la creación de elementos cerámicos. ${ }^{7}$ La organización del trabajo forma una parte importante de la logística del taller alfarero, y cuenta con funciones como la armonía y el sentido de jerarquía y autoridad en la toma de decisiones (Deal, 1998: 27).

La especialidad de don Emilio y su esposa Margarita es la creación de ciertos artefactos, como apastes, cajetes, tinajas e incensarios, figurillas y otros elementos. El hijo de don Emilio es Virgilio Espadas, ${ }^{8}$ quien a través del conocimiento aprendido heredó técnicas en la manufactura de los aerófonos de Juanita Dzul, que le permiten materializar instrumentos musicales, siendo él quien genera el mayor índice de manufactura de tales artefactos.

Cada alfarero obtiene una especialidad con la materia prima; de ésta podrá desarrollar una facilidad en la producción de los objetos, y también tendrá la habilidad técnica de creación y acabado en sus piezas. London (1991: 189) y Deal (1998: 35) infieren que el especialista posee una afiliación directa, con la que produce y mantiene un conocimiento de la materia prima, las técnicas y acabados de su rango de especialidad. El caso de Uayma es comparable a los citados, ya que los alfareros vigentes tienen una afiliación hacia tres tendencias, al reproducir artefactos: ${ }^{9}$

a) Alfarería doméstica, que se caracteriza por la producción de elementos como apastes, tinajas, cajetes, platos y otras piezas, como tortilleros; toda esta gama comprende artefactos de uso práctico empleados en una unidad doméstica.

b) Artesanal de venta; se distinguen por ser elementos que recrean visualmente la vida cotidiana y el entorno, en ellos se aprecia el valor social que cada consumidor se lleva, cada pieza es de carácter exclusivo y va desde

${ }^{7}$ Don Emilio es el sobrino directo de Juanita; tras la muerte de esta mujer, él emprende el trabajo del barro con los conocimientos aprendidos. En la línea de organización actual del taller, don Emilio es la cabeza y de él se desprenden su hijo Virgilio, su esposa Margarita y su sobrino Rafael.

${ }^{8}$ Joven alfarero, hijo de don Emilio; anualmente lleva a cabo un pequeño taller didáctico para niños donde enseña el arte de modelar en barro.

${ }^{9}$ Caso similar se presenta en el estudio de Deal (1998: 77). Él hace mención de estas tres tendencias que, de igual forma, son empleadas por los alfareros del área tzeltal. 
figurillas, ornamentación y artefactos como aerófonos. Para el alfarero de Uayma toda pieza es de venta y es de característica exclusiva y única.

c) Especializada; esta última se hace más evidente en la creación de aerófonos y figuras con un fin determinado, como figurillas de aluxes, ${ }^{10}$ aerófonos, tambores, y ornamentos de encargo especial.

\section{Áreas de producción}

Las modificaciones sociales y culturales también juegan un rol importante en la producción (Arnold, 2000). Las relaciones llevadas a cabo en todo espacio, tanto social como de producción, son vínculos que van más allá de lo técnico, conjeturas que a su vez implican una dialéctica, costumbres, tradiciones, religión, política y economía. Los espacios para la extracción de materia prima son ubicados en áreas favorables y cercanas, así como óptimas para el traslado y la facilidad logística de los artesanos. Otros elementos involucrados a la zona de estudio son la unidad habitacional, el taller, los hornos, las áreas de desecho, el espacio de preparación del material y la cavidad artificial que funge como un sistema de resguardo del mismo (Figura 2).

\section{Herramientas}

El material técnico para la creación de las piezas es conocido como instrumentos de trabajo, y con éstos se llega a la facilidad de las labores de producción (Torres y Rodríguez, 1991: 27). Con relación a la actividad que se desee realizar se ejercerán diversos medios de trabajo; un caso similar lo proporciona Deal (1998: 44) en los pueblos tzeltales donde las herramientas son diversas de acuerdo con las especificaciones particulares de cada vasija. Al nombrar los instrumentos de trabajo habrá que recordar que la alfarería se encuentra en una transición constante que ha ido modificando su tecnología (Deal, 1998: 36; Morales, 2005: 127).

Para la obtención de material en Uayma, se usan las palas, picos y barretas de metal y madera, además de elementos contenedores como cubetas. Durante el proceso de molienda se utiliza el metate o el molino eléctrico, de acuerdo a lo que la pieza específica requiera. Para la preparación de los materiales se requiere indumentaria práctica y cómoda para el alfarero. En esta localidad documentamos los siguientes elementos: para el trabajo de la arcilla se utilizan tambos, piletas, picoletas, palas, cubetas, metates, molino de mano, cestas y fibras para cernir el

\footnotetext{
${ }^{10}$ En la mitología maya estos seres son los encargados de velar por las milpas, y se representan por medio de figurillas de barro que son vueltas a la vida con una ceremonia ritual a cargo de jmen o chaman para fungir como guardianes de dicho espacio.
} 


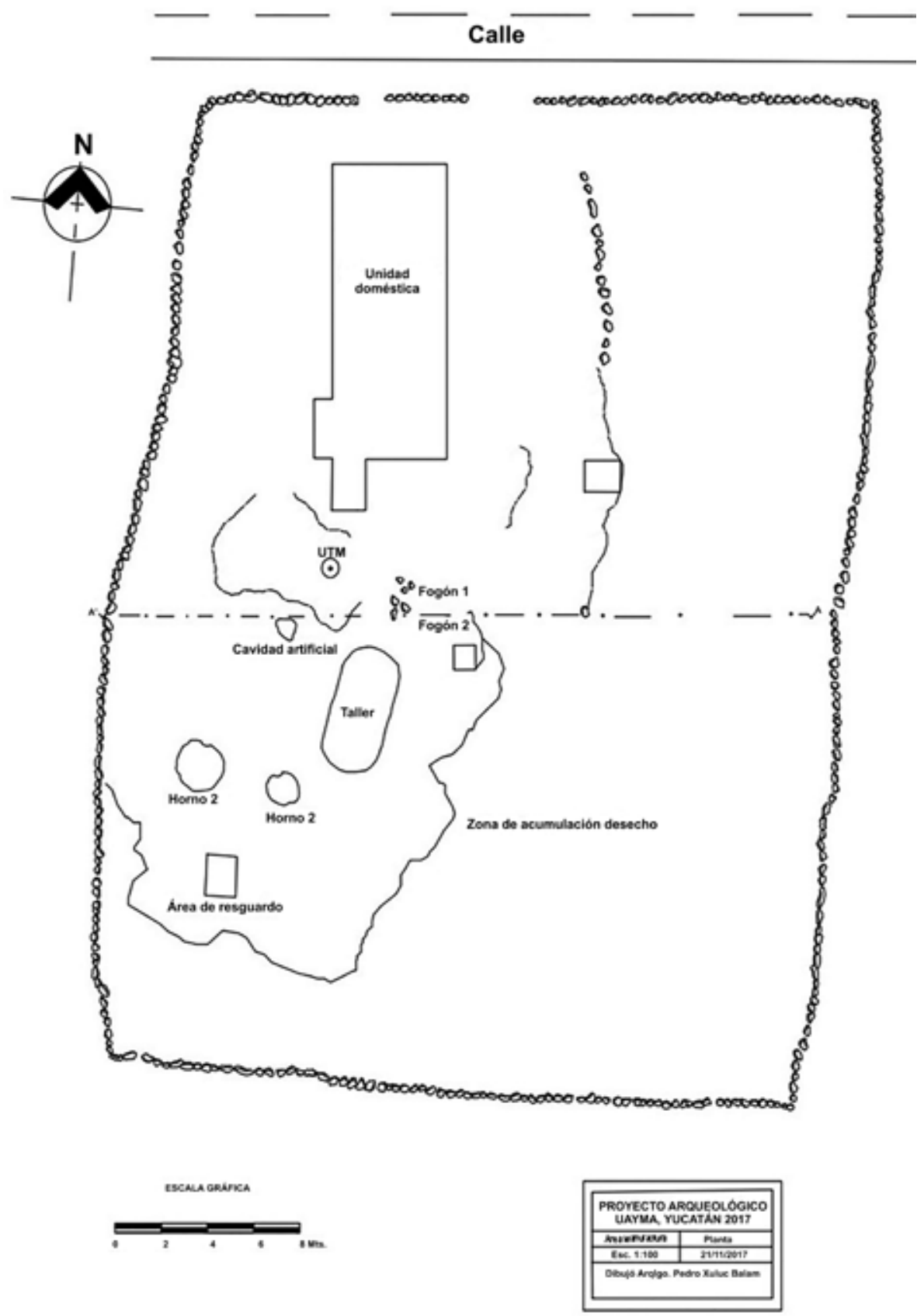

Figura 2. Plano del área de actividad de la familia alfarera Espadas Dzul en Uayma (Dibujo de Pedro Xuluc). 
material; para la manufactura de piezas, el torno, el k'abal, ${ }^{11}$ la jícara (luch), cuero, lijas, cuchillos y herramientas punzocortantes de material perecedero, como serían ramas diseñadas en formas puntiagudas (polib che), y un banquillo, además de moldes, piedras de bruñido y fibras de soskil (Xuluc, 2019a) (Figura 3).

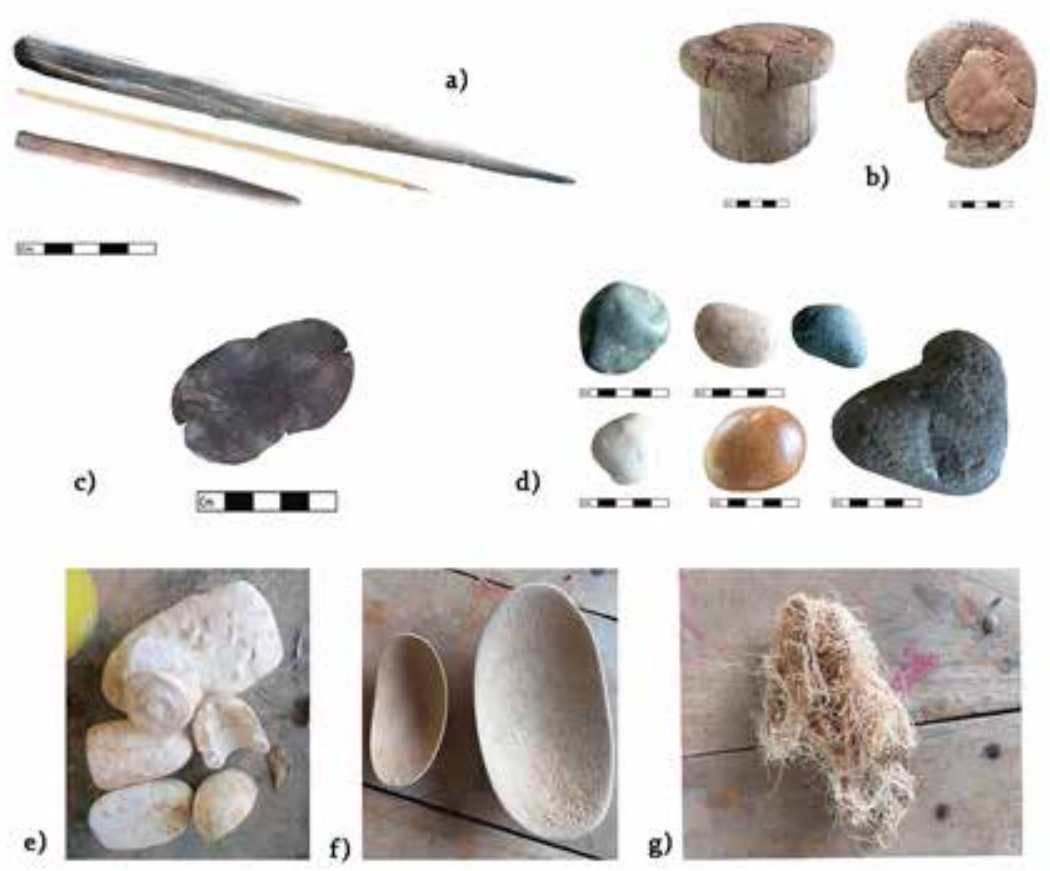

Figura 3. Indumentaria, a) polib che, b) torno antiguo / $\mathrm{kabal}$, c) cuero, d) diferentes piedras de bruñido, e) moldes, f) jícara, g) fibra de soskil (Fotografías de Pedro Xuluc).

\section{Aerófonos de Uayma, Yucatán}

En Uayma, entre los artefactos realizados se encuentran platos, cajetes, cazuelas, tinajas, comales, elementos con características de ornamentación, figurillas e instrumentos musicales. Específicamente, para este estudio se decidió generar un mejor panorama de los aerófonos, clasificados como silbatos y ocarinas.

Los aerófonos se distinguen por ser silbatos, ocarinas y flautas; éstos producen el sonido a partir de la oscilación de una masa de aire contenida en una cámara (Hornbostel y Sachs, 1961: 24). El sonido se genera cuando la corriente de aire proveniente de la boca del ejecutante incide en el bisel de la boca del

11 Torno hecho de madera y usado con los pies; sobre su base superior se dispone el barro a ser modelado. 
aerófono, la embocadura es un orificio que puede ser de forma circular/cuadrangular/rectangular, a través de la cual el aire del exterior pasa al interior de la cámara (Pacheco y Sánchez, 2011) y produce resonancia que puede ser o no ser regulada por orificios de digitación.

Las características de los elementos siempre connotan una relación con la especialidad que se tiene en su creación. El instrumental sonoro se vincula con la forma de la pieza, su acústica y las tonalidades de alcance que pueda lograr; de acuerdo con lo planteado por diversos estudios, los silbatos se caracterizan por ser generadores de sonido en movimiento, no poseen agujeros y pueden tener o no cámaras divisorias (Zalaquett, Sierra y Jiménez, 2013: 35); sin embargo, en Uayma los silbatos y flautas son concebidos con orificios de digitación, al igual que las ocarinas. Su manufactura se realiza a partir del modelado a mano, la incisión y el pastillaje; en algunos casos se presentan elementos elaborados con moldes, pero son una fracción mínima de los producidos. Entre sus rasgos generales son elaborados con la técnica del ahuecado y pueden o no agregarles elementos suntuarios o algún tratamiento previo a la cocción o postcocción, como la pigmentación de la pieza.

Los aerófonos creados en el taller de Uayma son clasificados como silbatos y ocarinas, y representan figuras antropomorfas y zoomorfas; entre ellas se resalta la creación de aves, mamíferos, reptiles, cabezas humanas y elementos biofísicos representativos del hombre, como rostros o cráneos. En su mayoría, cada aerófono es diseñado de forma exclusiva y única; se modela de acuerdo con lo que el alfarero perciba e imagine (Figura 4). El medio y su entorno social forman parte esencial del sonido que se quiera producir; si el artesano gusta de las codornices (Colinus nigrogularis), ${ }^{12}$ hará lo necesario para su reproducción en cuanto al sonido y forma.

Entre las especies representadas se encuentran las palomas de monte o de ala blanca Zenaida asiatica (sac pakal), el cenzontle Mimus gilvus (chiká), el colibrí Doricha eliza (dzunum), los gallos y gallinas Gallus domesticus (kax), la tórtola rojiza Columbina talpacoti (mucuy), los patos Anas sp. y el zanate Quiscalus mexicanus $\left(x^{\prime} k a u\right)$. Con respecto a los mamíferos que se modelan se tiene el perro Canis familiaris (peek), el jaguar Panthera onca (balam) y el venado cola blanca Odocoileus virginianus (ceh), así como la corzuela de Yucatán, Mazama pandora (yuk). Entre los reptiles se tienen en el modelado a las tortugas, Terrapene sp. (áak), y anfibios, como ranas ${ }^{13}$ (muuch).

Cada pieza presenta atributos únicos en su producción, factores que van desde la concepción ideotécnica de su creación hasta elementos físicos que modifican su sonido y acústica. El instrumental sonoro producido en el taller se puede

\footnotetext{
${ }^{12}$ Los nombres científicos de la fauna fueron tomados de $<$ www.biodiversidad/enciclovida.org. $\mathrm{mx}>$.

${ }^{13} \mathrm{Al}$ ser varias las especies que se encuentran en el estado no se da una especificación de la que se representa (ver García, 2015).
} 


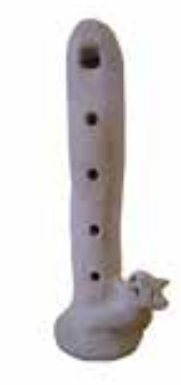

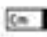
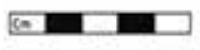

$5=$
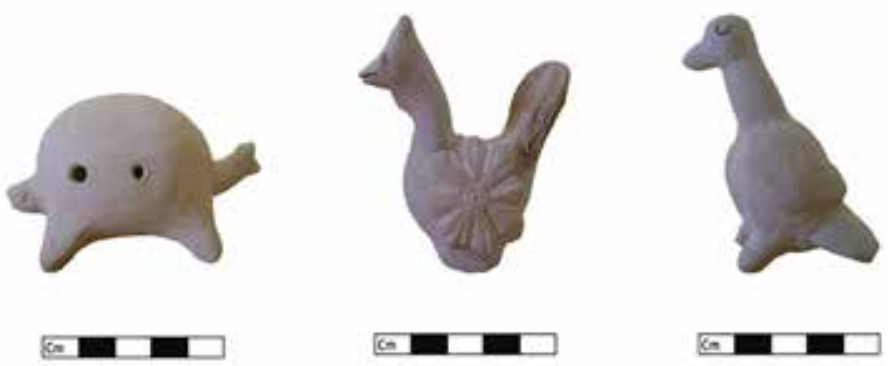

Figura 4. Aerófonos elaborados en Uayma, Yucatán, de derecha a izquierda: flauta, ocarina y silbatos

(Fotografía de Pedro Xuluc).

representar en diversas formas, unas antropomorfas y otras, como se mencionó anteriormente, zoomorfas. Estos objetos son manufacturados con una mezcla específica de pasta, que contiene elementos distintos a los que presentan otros artefactos manufacturados en la comunidad (ver Tabla 1).

\begin{tabular}{|c|c|c|}
\hline PROPIEDAD & ELEMENTOS & MEZCLA $^{*}$ \\
\hline $\begin{array}{c}\text { Elementos contendores } \\
\text { de líquido }\end{array}$ & Tinajas, cántaros y vasos & $\begin{array}{c}\text { Barro + y'ak'aat + tierra roja } \\
(\text { kankaab) }\end{array}$ \\
\hline Resistencia & Cajetes, comales & Calcita (j'ib), pickich y barro \\
\hline Elementos sonoros & $\begin{array}{c}\text { Silbatos, ocarinas, } \\
\text { piezas de percusión }\end{array}$ & $\begin{array}{c}\text { Sahcab, calcita (j'ib), tierra roja } \\
\text { (kankaab) y barro }\end{array}$ \\
\hline
\end{tabular}

Tabla 1. Mezclas en la pasta empleadas en la producción alfarera de Uayma ("La cantidad requerida en cada mezcla será equivalente a lo que se desee manufacturar).

El color de los aerófonos puede ser decidido por el maestro alfarero o por el productor, y entre la gama de pigmentos se tienen: crema, anaranjado, bayo, el rojo oxido, a base de tierra roja, y la coloración a través de pinturas de cal (Figura 5). Los elementos diseñados para un fin específico, como el musical, pueden o no presentar decoración externa. La complejidad de la manufactura dependerá de las características internas y externas, la variante acústica y el objetivo de uso. 


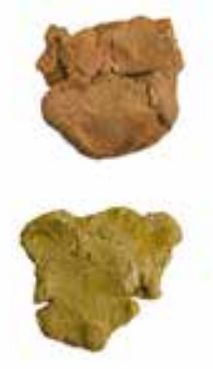

a)
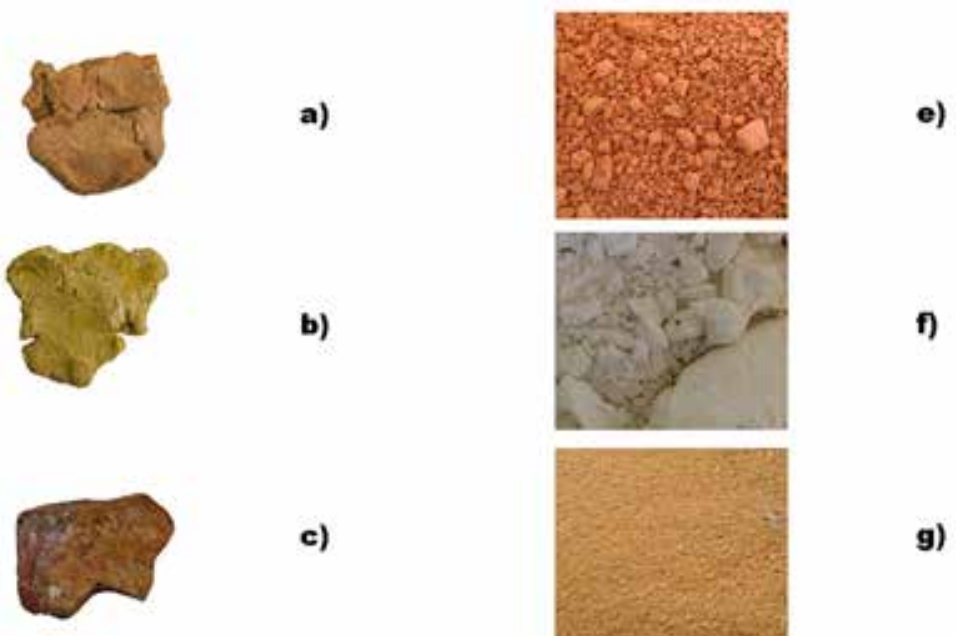

b)

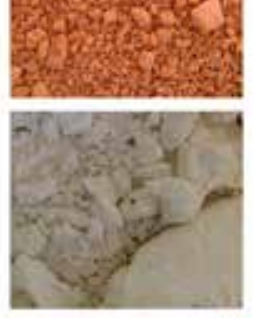

e)

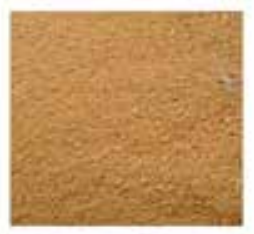

f)

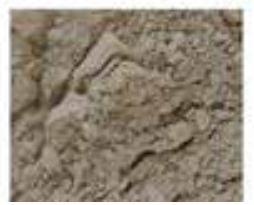

g)

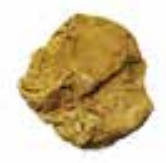

d)

\section{h)}

Figura 5. Materiales agregados en la manufactura cerámica: a), b), c),

d) arcillas usadas en la producción alfarera uaymense, e) tierra roja conocida como pickich, f) material pétreo granulado, g) tierra roja kankaab, h) barro molido

(Fotografías de Pedro Xuluc).

Desde la década de 1960 está documentado el uso de técnicas de imagenología en arqueología, primero con la aplicación de rayos X al estudio de restos óseos, posteriormente la tomografía axial computarizada (TAC) y, recientemente, la resonancia magnética (RM) (Lantes y Prieto, 2017; Flores y Flores, 1981). En esta investigación se decidió emplear la técnica de análisis por radiografía con el objetivo de apreciar las características en la morfología interna de ocarinas y silbatos manufacturados actualmente en la mencionada comunidad de Uayma.

Los parámetros para el estudio plantearon entender y comparar seis piezas de barro desde las perspectivas de creación de un alfarero especializado en la producción de instrumentos sonoros y de otro que no fuese especialista; estos seis elementos corresponden a una acotada muestra de un conjunto mayor que constaba de doce elementos (Xuluc, 2019b). Con tales análisis se busca establecer puentes que enlacen los datos etnográficos con los técnicos, que comúnmente se suelen obtener en el campo arqueológico y análisis de material, aportando una propuesta de interpretación que se sume a las ya existentes para la clasificación, distinción y registro de las piezas de esta clase en arqueología. 


\section{Resultados}

Aerófonos XM-2 y XM-10

Las condiciones prevalentes en estas piezas son diferenciadas en sus estilos de manufactura, y aunque ambas corresponden a la clasificación de aerófonos presentan variantes que las distinguen. En este caso el pensamiento de dos alfareros en sus diversas concepciones tanto organológicas, sociales y de conocimiento particular en manufactura se concretan al plasmar físicamente a un jaguar.

La ocarina XM-2 evidencia un trabajo práctico y sencillo de un instrumento sonoro que plasma en sus paredes un conocimiento homogéneo del modelado, con una estética simple sin considerable detalle (Figura 6). El orificio se hizo en la parte superior de la espalda del jaguar y su canal de insuflación pegado al lateral del mismo; estos son indicios de una manufactura poco especializada del elemento, ya que ello generará un corto alcance sonoro y poca comodidad de uso.

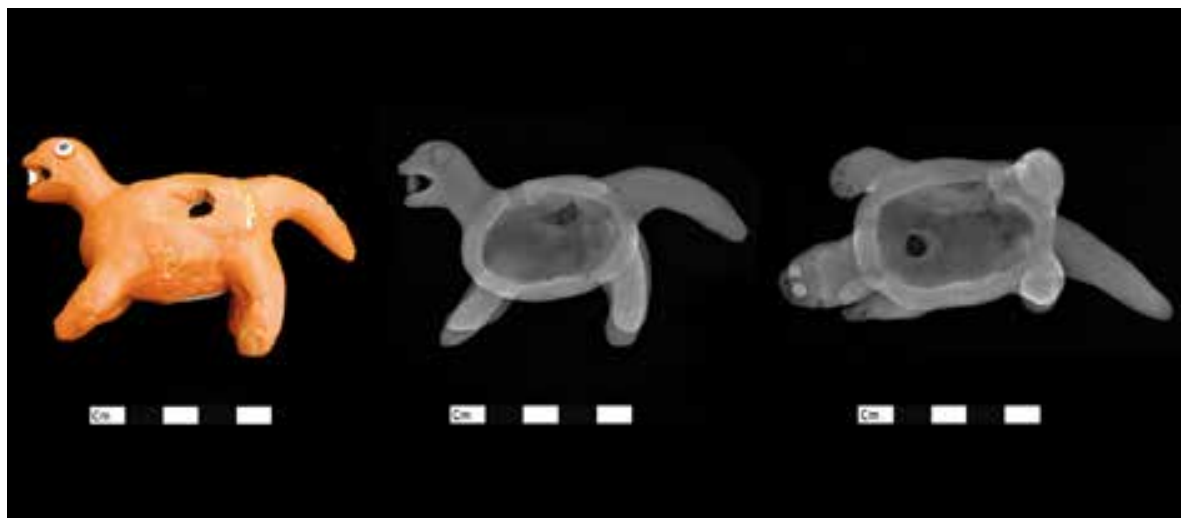

Figura 6. Ocarina XM-2 (Fotografías de Pedro Xuluc).

Por su parte, la pieza XM-10 es una ocarina manufacturada por el artesano especialista; en sus condiciones organológicas se integra una mejor disposición del conocimiento práctico de ejecución y una calidad sonora mayor a la de la ocarina XM-2 (Figura 7). En su parte posterior se encuentra el canal de insuflación, el cual fue manufacturado en forma alargada, generando una mejor condición de ejecución y una mayor escala de aire que pasará a la cámara de resonancia. La manufactura de la cámara en su morfología interna presenta una forma burda; estéticamente no se distingue una especialización, pero las paredes de la cámara fueron pensadas a propósito para que el aire que penetre tenga una distorsión y el sonido presente características diversas. Esta ocarina (XM-10) evidenció dos orificios de escape, los cuales fueron plasmados con medidas diferentes, y la intención del alfarero fue confeccionar tonalidades desiguales en la pieza. 


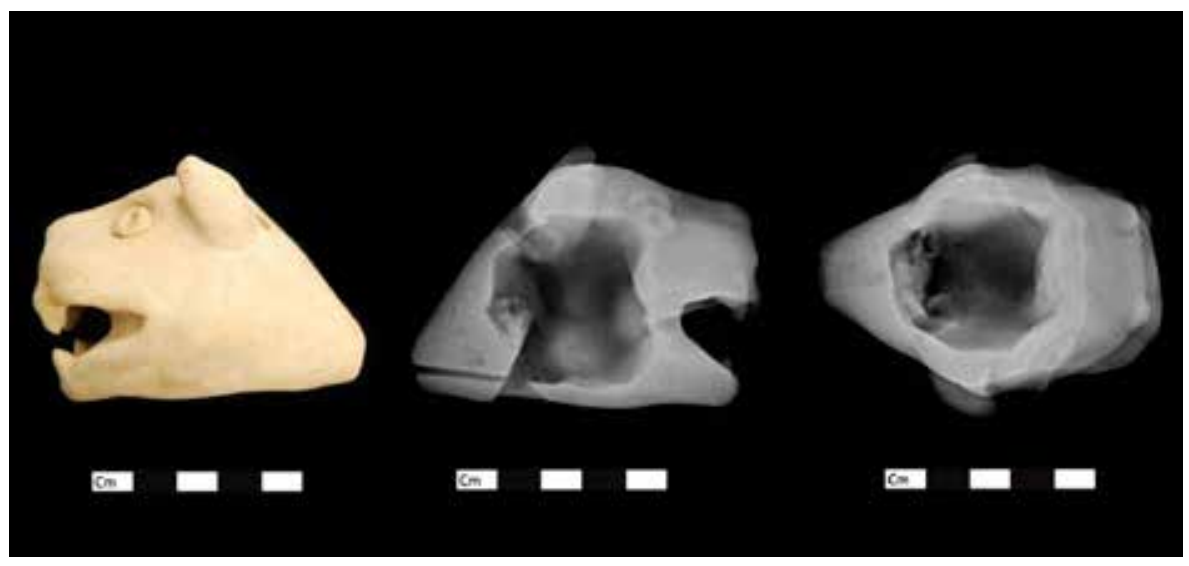

Figura 7. Ocarina XM-10 (Fotografías de Pedro Xuluc).

Estas piezas son un claro ejemplo de las condiciones prevalentes de manufactura entre un alfarero especialista y otro que no lo es.

\section{Ocarinas $X M-4$ y $X M-12$}

El siguiente elemento trata de un reptil, una tortuga. Estos artefactos son determinados en su uso por las características de especialización y uso recreativo.

La ocarina XM-4 presentó entre sus rasgos una forma estilizada, precisión y nitidez sonora al ejecutarse, además de su reducido tamaño. A pesar de ser una pieza estéticamente idónea en su manufactura, tanto en su morfología interna como externa, la ocarina está destinada a un empleo recreativo ya que sus niveles sonoros nos son ideales para la ejecución (Figura 8).

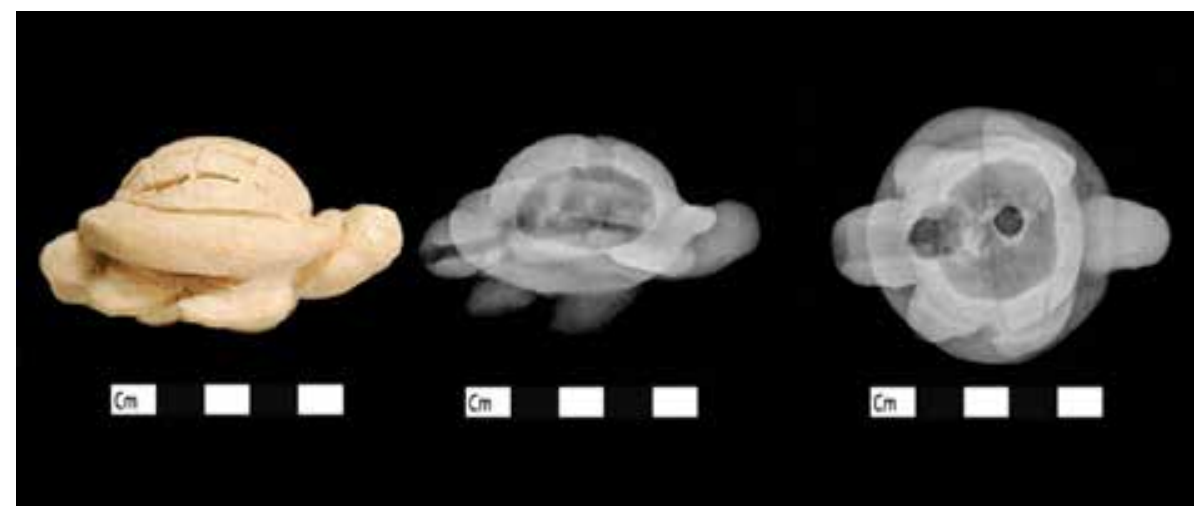

Figura 8. Ocarina XM-4 (Fotografías de Pedro Xuluc). 
Por otra parte, el elemento denominado XM-12 presentó rasgos que en su morfología interna y externa denotan una habilidad mayor de manufactura y disposición para su uso específico. En su modelado externo tiene características estéticas óptimas que lo hacen sobresalir a la vista, su abultado caparazón que encierra la cámara de resonancia es parte de estos rasgos físicos y sonoros (Figura 9).

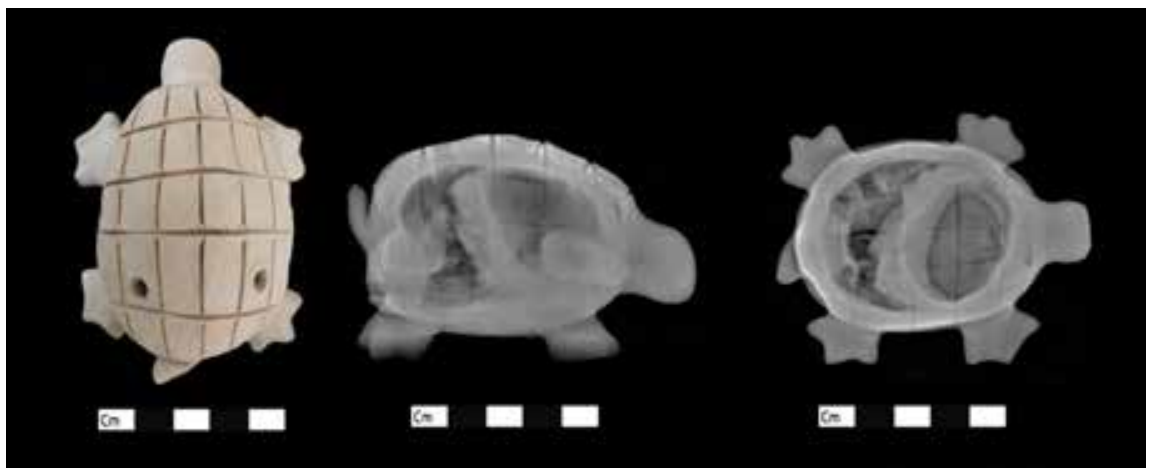

Figura 9. Ocarina XM-12 (Fotografías de Pedro Xuluc).

Al interior, la cámara se encontró dividida en dos apartados; el primero se establece en la parte superior de la pieza y fue determinado por paredes claras y definidas; el segundo, en la parte posterior, presenta paredes burdas poco definidas; el paso del aire es a través del aeroducto que está confeccionado en el costado izquierdo al interior de la cámara; este ducto es el encargado del paso de aire a través de ambas cámaras internas; los orificios de digitación se ubican en la parte superior del caparazón y son uniformes; estos orificios se establecen entre ambas cámaras internas generando de tal manera una variante acústica en la pieza.

\section{Ocarinas XM-7 y XM-8}

En la siguiente comparación se abordan dos muestras con rasgos antropomorfos; en ambos casos se trataron de ocarinas; en ellas se plasman las intenciones ideotécnicas que rigen la concepción de dos estados anímicos del ser, la vida y la muerte, en específico en la ocarina XM-8.

El elemento XM-7 se relaciona con actividades o usos recreativos. Las calidades internas de las paredes evidencian un trabajo definido pero sencillo; es decir, sin contar con una filiación a la manufactura de aerófonos especializados; caso similar al observado en el aerófono XM-2, donde el trabajo práctico en la confección de un instrumento sonoro plasma un conocimiento homogéneo del modelado (Figura 10). Sus características externas evidencian una rica estética en los atavíos, como orejeras, tocado y rasgos fisonómicos. 


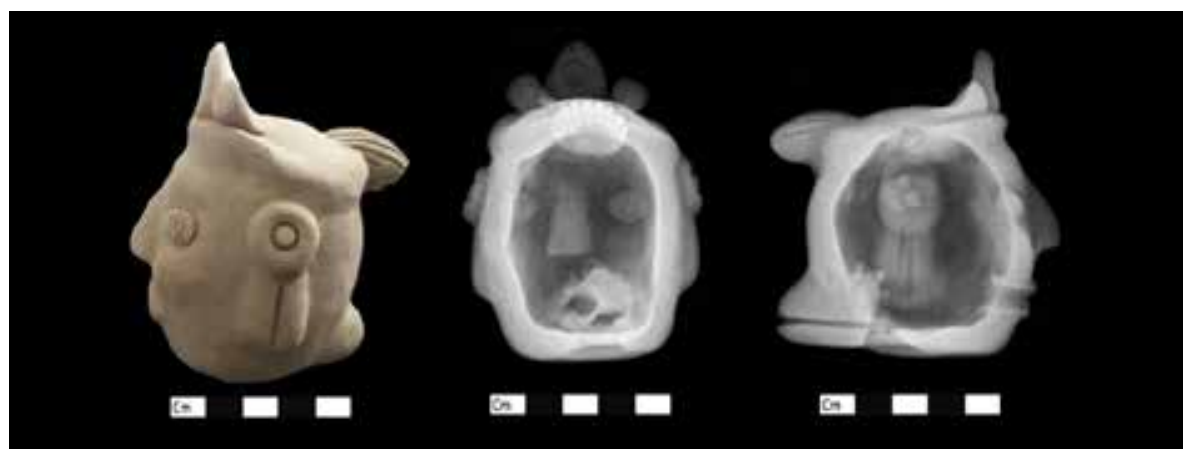

Figura 10. Ocarina XM-7 (Fotografías de Pedro Xuluc).

A su vez, la ocarina XM-8 presentó las características de ser una figura antropomorfa con rasgos masculinos; también representa un nexo entre vida y muerte, tanto físicamente al plasmarse en la pieza y como agente simbólico inmerso en el sentido anímico expresado por el alfarero, así como por la asociación de lo anterior con su frecuencia sonora (Figura 11).

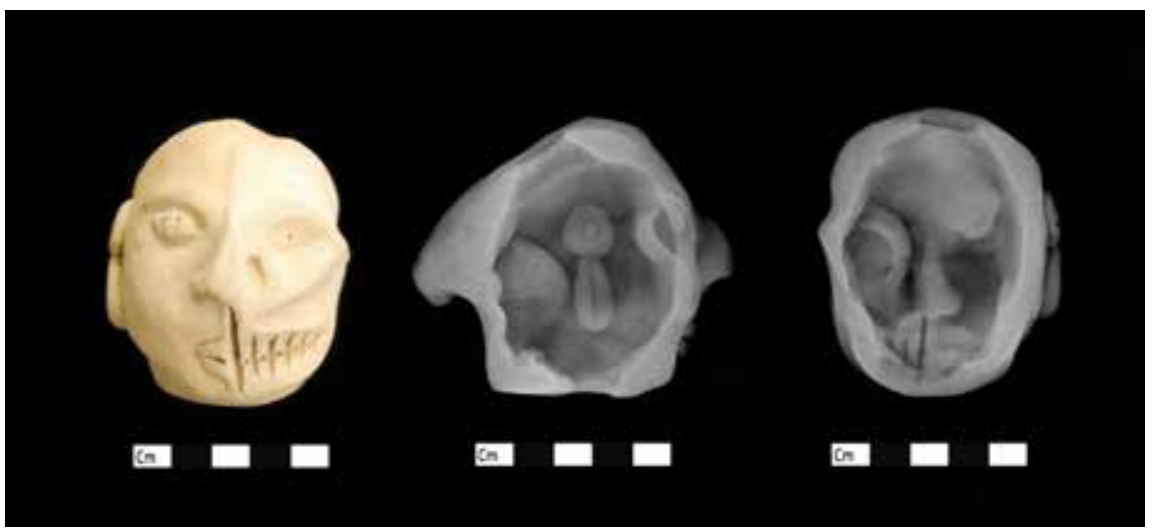

Figura 11. Ocarina XM-8 (Fotografías de Pedro Xuluc).

Entre sus cualidades internas se observó una definición "tosca” y un espesor delgado en las paredes de la cámara de resonancia; el aeroducto es directo y su ejecución es a través del soplado, pegando la boca al orificio y tapando con la mano el aeroducto de escape que se encuentra en la parte inferior de la pieza. Entre sus rasgos externos muestra atavíos en las orejas y la fisonomía marcada propia de los habitantes de Yucatán; también se notó la intención de generar la comodidad en su ejecución al alargar la agarradera ubicada en el parietal del artefacto. En esta pieza se aprecia un simbolismo que enmarca dos fases de 
tiempo; esto se recrea también en la calidad del sonido. El artefacto presentó un sonido opaco, "seco" y grave durante su ejecución, haciendo alusión al sentido fúnebre o turbio del concebir la fase mortuoria; este aspecto fue intencional y fue compartido por el artesano durante la recolección de datos.

\section{Conclusiones}

La documentación de las secuencias de trabajo de los alfareros nos hace entender la complejidad que implica el manufacturar elementos sonoros (García, 2015; Xuluc, 2019b); en este caso, los aerófonos son más que simples objetos, ya que involucran una agencia que se relaciona con el espacio de manufactura, la tecnología de creación y las concepciones ideotécnicas (Parrales, 2017).

En relación con los aerófonos, el haber entendido las morfologías interiores y exteriores de algunas de estas piezas es una forma de conocer y generar aproximaciones que ofrezcan un panorama amplio para un tipo de artefactos que pudieron ser parte importante de dinámicas sociales en el pasado. Las piezas analizadas tras la imagenología de rayos X evidencian la especialización, tecnología y distinción a través de las comparaciones en los artefactos producidos, en los cuales se pudo observar lo siguiente:

- Las paredes de los aerófonos son distintivas, es decir, se caracterizan por ser delgadas, midiendo entre $2 \mathrm{~mm}$ y $4 \mathrm{~mm}$; esta distinción es generada tras el conocimiento de juego del sonido en relación con la cámara de resonancia y el canal de insuflación. Si una pieza presenta paredes gruesas pierde resonancia y alcance.

- Un aerófono fabricado por un especialista tiene una mayor complejidad intencional; es el caso de los aerófonos XM-4 y XM-12, donde el primero fue manufacturado por un artesano no especialista y presentó una mejor estética en su composición interna, pero no mostró una manufactura acorde a un uso sonoro.

- La decisión estética de la pieza puede ser variada; el uso lo designa la propiedad de la morfología interna que hará variar las cualidades sonoras que ésta manifieste.

- Las herramientas de trabajo son las encargadas de la acción física en la especialización y creación de piezas sonoras; de ellas depende un empleo óptimo para la manufactura de orificios, canales de insuflación y demás características de los aerófonos. Si éstas son mal empleadas, las propiedades de la pieza no serán óptimas para la ejecución y reproducción del sonido. 
Aunque en el presente trabajo ejemplificamos una muestra acotada, a través de comparaciones analizadas se han podido distinguir características pocas veces percibidas en el artefacto y, de acuerdo a éstas, entender las intenciones de su funcionalidad y de su manufactura. Tales elementos son sólo una pequeña muestra de otras piezas que ejemplifican la producción de aerófonos en la alfarería peninsular yucateca.

\section{Agradecimientos}

Al Laboratorio de radiología de la Clínica de Especialidades Médicas Santa María S.A. de C.V., así como a la familia alfarera Espadas Dzul de la comunidad de Uayma.

\section{Bibliografía}

Andrews, Edward Wyllys

1973 Flautas precolombinas procedentes de Quelepa, El Salvador. San Salvador: Ministerio de Educación, Dirección de Publicaciones (Colección Antropología).

Arnold, Philip. J., III

2000 "Working Without a Net: Recent Trends in Ceramic Ethnoarchaeology", Journal of Archaeological Research, VIII (2): 105-133. Dol: https://doi. org/10.1023/A:1009452310915.

Barrera Vásquez, Alfredo (coord.)

1980 Diccionario maya-español, español-maya. Mérida: Cordemex.

Castillo Aguilar, Víctor, Héctor Neff, Ronald Bishop, Erin L. Sears y James Michael

2009 "Mujeres y contrahechos: las figurillas moldeadas de la Costa Sur de Guatemala", XXII Simposio de Investigaciones Arqueológicas en Guatemala, pp. 967985, Juan Pedro Laporte, Bárbara Arroyo y Héctor Mejía (eds.). Guatemala: Museo Nacional de Arqueología y Etnología.

Comisión Nacional para el Conocimiento y Uso de la Biodiversidad

$2019<$ http://www.biodiversidad/enciclovida.org.mx > [consultada el 22 de mayo del 2019].

Costin, Cathy Lynne

1991 "Craft Specialization: Issues in Defining, Documenting, and Explaining the Organization of Production", Archaeological Method and Theory, III: 1-56.

2001 "Craft Production Systems", Archaeology at the Millenium: A Sourcebook, pp. 273-327, Gary M. Feinman y Douglas Price (eds.). Nueva York: Kluwer Academic, Plenum Publishers. 
Deal, Michael

1983 "Pottery Ethnoarchaeology among the Tzeltal Maya", tesis de Doctorado en Filosofía. Columbia: Universidad Británica de Burnaby, Departamento de Arqueología.

1998 Pottery Ethnoarchaeology in the Central Maya Highlands. Salt Lake City: University of Utah Press.

Flores Dorantes, Felipe y Lorenza Flores García

1981 Organología aplicada a instrumentos musicales prehispánicos: silbatos mayas de México. México: Instituto Nacional de Antropología e Historia, Museo Nacional de Antropología.

García Magdaleno, Pavel Alonso

2015 "El simbolismo de ranas y sapos en el oriente de Yucatán”, tesis de maestría en Estudios Mesoamericanos. México: Universidad Nacional Autónoma de México, Facultad de Filosofía y Letras.

Gosselain, Olivier P. y Alexandre Livingstone Smith

2005 "The Source. Clay Selection and Processing Practices in Sub-Saharan Africa", Pottery Manufacturing Processes: Reconstitution and Interpretation, pp. 65-82, Alexandre Livingstone Smith, Dominique Bosquet y Remi Martineau (eds.). Oxford: BAR (BAR International Series, 1349).

Hernández Fajardo, José

1977 "Historia de las artes menores", Enciclopedia Yucatanense, tomo IV, pp. 823914. Mérida: Gobierno de Yucatán.

Hodder, Ian

1991

"Interpretive Archaeology and Its Role", American Antiquity, 56 (1): 7-18. Dol: https://doi.org/10.2307/280968.

Hornbostel, Erich von y Curt Sachs

1961 “Classification of Musical Instruments", The Galpin Society Journal, 14: 3-29. Dol: https://doi.org/10.2307/842168.

Jover Maestre, Francisco Javier

1999 Una nueva lectura del "Bronce Valenciano". Alicante: Universidad de Alicante.

Lantes Suárez, Oscar y Pilar Prieto Martínez

2017 "Técnicas de imagen de rayos X y arqueología. Estado de la cuestión y potencialidad de la técnica", Antrope, VII: 14-36.

London, Gloria A.

1991 "Standardization and Variation in the Work of Craft Specialist", Ceramic Ethnoarchaeology, pp. 182-204, William A. Longrace (ed.). Arizona: University of Arizona Press.

Mata Amado, Guillermo

2006 "Silbatos prehispánicos de la Costa Sur del departamento de Santa Rosa, 
Guatemala”, XIX Simposio de Investigaciones Arqueológicas en Guatemala, 2005, pp. 189-199, Juan Pedro Laporte, Bárbara Arroyo y Héctor Mejía (eds.). Guatemala: Museo Nacional de Arqueología y Etnología.

Morales Valderrama, Carmen

1992 "Cinco artesanías del oriente de Yucatán, alfarería, bordados, cestería, joyería y talabartería”, Cuadernos de la cultura yucateca, 1: 1-9.

2005 "La alfarería de Yucatán: una tradición al finalizar el siglo xx”, La producción alfarera en el México antiguo, I, pp. 121-142, Leonor Merino Carrión y Ángel García Cook (coords.). México: Instituto Nacional de Antropología e Historia.

Orton, Clive

1997 La cerámica en arqueología. Barcelona: Grijalbo Mondadori.

Pacheco Silva, Mónica y Gonzalo Sánchez Santiago

2011 "Los instrumentos musicales mayas en el Museo de Etnología de Hamburgo", XXIII Simposio de Investigaciones Arqueológicas en Guatemala, 2010, pp. 909-922, B. Arroyo, L. Paiz, A. Linares y A. Arroyave (eds.). Guatemala: Museo Nacional de Arqueología y Etnología.

Parrales García, María Fernanda

2017 "El imaginario de los sonidos: aerófonos figurados del Valle de Oaxaca. De lo prehispánico a lo contemporáneo", tesis de maestría en Historia del Arte. México: Universidad Nacional Autónoma de México, Facultad de Filosofía y Letras, Instituto de Investigaciones Estéticas.

Polanyi, Karl

1968 "The Economy as an Instituted Process”, Economic Anthropology: Readings in Theory and Analysis, pp. 122-142, Edward E. LeClair, Jr. y Harold Schneider (eds.). Nueva York: Holt, Rinehart and Winston.

Rendón, Silvia

1947 “Notas sobre la alfarería indígena de la península de Yucatán”, Revista Mexicana de Estudios Antropológicos, IX (1-3): 107-123.

Rice, Prudence

1996 "Recent Ceramic Analysis: 2. Composition, Production, and Theory", Journal of Archaeological Research, 4: 165-202. Dor: http://doi-org-443.webvpn. fjmu.edu.cn/10.1007/BF02228880.

Rosales González, Margarita y Amada Rubio Herrera

2010 "Apicultura y organizaciones de apicultores entre los mayas de Yucatán", Estudios de Cultura Maya, XXXV: 163-186. DoI: http://dx.doi.org/10.19130/iifl. ecm.2010.35.27.

Sillar, Bill

2000 Shaping Culture. Making Pots and Constructing Households, Ethnoarchaeological Study of Pottery Production, Trade and Use in the Andes. Oxford: J. and E. Hedges, Archaeopress. 
Sinopoli, Carla M.

1991 Approaches to Archaeological Ceramics. New York: Springer Science \& Business Media.

Terán, Silvia y Christian Rasmussen

1981 Artesanías de Yucatán. Mérida: Secretaría de Educación Pública, Arte y Comunicación, Dirección General de Culturas Populares.

Thompson, Raymond

1958 "Modern Yucatan Mayan Pottery Making", American Antiquity: Memoirs of the Society for American Archaeology, XXIII (4): 157.

Torres Quintero, Sergio y Catalina Rodríguez Lazcano

1991 Alfarería maya de tierras bajas. México: Instituto Nacional de Antropología e Historia.

Varela Torrecilla, Carmen

1990 "La producción alfarero-artesanal del occidente de la península de Yucatán: un ejemplo de cambio cultural”, Revista Española de Antropología Americana, XXIII: 83-100.

Xuluc Balam, Pedro Rogelio

2019a "Un estudio etnoarqueológico a la producción cerámica en la comunidad de Uayma, Yucatán. El caso de la extracción de materia prima”. Informe de actividades. Archivo del Centro InAH Yucatán.

2019b "Xoob Meyaj: la producción de aerófonos en la comunidad de Uayma, Yucatán. El caso de silbatos y ocarinas", tesis de licenciatura en Arqueología. Mérida: Universidad Autónoma de Yucatán, Facultad de Ciencias Antropológicas.

Zalaquett, Francisca, Thelma Sierra y Socorro Jiménez

2013 "Sonidos y acciones rituales. Los instrumentos musicales del sitio arqueológico de Xcambó, Yucatán”, Continuidad, cambios y rupturas en la religión maya, pp. 17-57, Mercedes de la Garza y Carmen Valverde (coords.). México: Universidad Nacional Autónoma de México, Instituto de Investigaciones Filológicas, Centro de Estudios Mayas.

Pedro Rogelio Xuluc Balam. Mexicano. Licenciado en Arqueología por la Universidad Autónoma de Yucatán. Es profesor investigador asociado del Centro INAH Yucatán. Sus especialidades son la etnoarqueología y la arqueología de patrón de asentamiento, el paisaje, la arquitectura y las unidades domésticas en el norte de Yucatán. Su investigación en curso se enfoca en el Cono Sur del estado de Yucatán.

royerterron@hotmail.com 